\title{
Transport Coefficients of Gluon Plasma from Lattice QCD
}

\author{
Sunao Sakai* \\ Yamagata University \\ E-mail: sakaide.yamagata-u.ac.jp
}

\section{Atsushi Nakamura}

RIISE Hiroshima University

E-mail: nakamura@riise.hiroshima-u.ac.jp

RHIC is now confirming the discovery of the "New State of Matter", whose properties are gradually revealed by experimental data. It is not the free gas state, but well described by a fluid with very small viscosity. It is now highly desired to calculate the value of the viscosity from the fundamental theory, i.e., QCD just above the transition temperature. In this report we present our calculation of the transport coefficient of gluon system on $24^{3} \times 8$ lattice in the quench approximation. Simulations are carried out in the range, $1.4 \leq T / T_{c} \leq 24$. In the temperature region slightly above the transition, where the perturbative calculation is not applicable, the shear viscosity is smaller than typical hadron masses. The bulk viscosity is consistent with zero within the range of error bars in $1.4 \leq T / T_{c} \leq 24$. We compare our results with the perturbative calculations in large $T / T_{c}$ region. It is found that the lattice and perturbative results are consistent with each other there. The ratio $\eta / s$ is around $0.1-0.4$ in $T / T_{c}<3$ region and satisfies the KSS bound. In order to estimate the contribution from high frequency part of the spectral function, we add a term, $\rho^{\text {high }}$, proposed by Aarts and Resco[3]. It is found that until the threshold mass becomes small, its effect is quite small, and that viscosity decreases as the threshold decreses. From these studies we think that although our result is obtained under an assumptions for the spectral function, it gives a reasonable estimation for $d \rho / d \omega$ at $\omega=0$, and qualitative results will not be changed when the accurate spectral function is obtained.

XXIIIrd International Symposium on Lattice Field Theory

25-30 July 2005

Trinity College, Dublin, Ireland

\footnotetext{
* Speaker.
} 


\section{Introduction}

The RHIC data are now revealing some properties of a "New State of Matter", which is expected to be quark-gluon plasma(QGP). The jet quenching data suggest that its mean free path is short, and the phenomenological analysis of the elliptic flow shows that, it could be explained by a fluid model with small viscosity. Namely it is almost perfect fluid. As the viscosity is proportional to mean free path[1], this analysis also indicates that it is short. These experimental data and phenomenological analysis show that the "New State of Matter" is a strongly interacting system, because mean free path is proportional to the inverse of the scattering cross section times density.

It is a natural anticipation that transport coeffi cients of the QGP must be smaller than a ordinary matter, like water or oil. Therefore it is now urgent to calculate transport coeffi cients from QCD. As the QGP near the transition temperature is strongly interacting, a non-perturbative calculation is indispensable. In Ref.[2] we have published our results from lattice simulations in the $1.5<$ $T / T_{c}<2.1$ region. In this report we study in higher temperature region, $T / T_{c}<24$, and compare our results with perturbative calculations. We also discuss how our results depend on the spectral function of the green function in the high frequency region[3].

On a lattice, calculation of the transport coeffi cients is formulated in the framework of the linear response theory [4, 5].

$$
\begin{gathered}
\eta=-\int d^{3} x^{\prime} \int_{-\infty}^{t} d t_{1} e^{\varepsilon\left(t_{1}-t\right)} \int_{-\infty}^{t_{1}} d t^{\prime}<T_{12}(\vec{x}, t) T_{12}\left(\overrightarrow{x^{\prime}}, t^{\prime}\right)>_{r e t} \\
\frac{4}{3} \eta+\zeta=-\int d^{3} x^{\prime} \int_{-\infty}^{t} d t_{1} e^{\varepsilon\left(t_{1}-t\right)} \int_{-\infty}^{t_{1}} d t^{\prime}<T_{11}(\vec{x}, t) T_{11}\left(\vec{x}^{\prime}, t^{\prime}\right)>_{r e t}
\end{gathered}
$$

where $\eta$ is shear viscosity, and $\zeta$, bulk viscosity. $\left\langle T_{\mu \nu} T_{\rho \sigma}>_{\text {ret }}\right.$ is a retarded Green's function of energy momentum tensor at given temperature. In the quenched model, it is written by the fi eld strength tensor as follows.

$$
T_{\mu \nu}=2 \operatorname{Tr}\left[F_{\mu \sigma} F_{v \sigma}-\frac{1}{4} \delta_{\mu v} F_{\rho \sigma} F_{\rho \sigma}\right]
$$

The fi eld strength tensor is defi ned by the plaquette operator, $L_{\mu v}(x)=\exp \left(i a^{2} g F_{\mu v}(x)\right)$.

The shear viscosity in Eq.1.1 is also expressed by using a spectral function $\rho$ of the retarded Green's function $\rho(\omega)[5]$ as follows.

$$
\eta=\pi \lim _{\omega \rightarrow 0} \frac{\rho(\omega)}{\omega}=\pi \lim _{\omega \rightarrow 0} \frac{d \rho(\omega)}{d \omega}
$$

For the determination of the spectral function $\rho(\omega)$, we use a well known fact that the spectral function of the retarded Green's function at temperature $T$ is same as that of Matsubara-Green's function. Therefore our target is to calculate Matsubara-Green's function $\left(G_{\beta}\left(t_{n}\right)\right)$ on a lattice and determine $\rho$ from it.

\section{Lattice calculation of $\eta$ from Matsubara-green's function $G_{\beta}(t)$}

In order to determine the spectral function $\rho(\omega)$ from $G_{\beta}\left(t_{n}\right)$, the most promising method may be the maximum entropy method(MEM). However to calculate $\rho(\omega)$ precisely by MEM, $G_{\beta}\left(t_{n}\right)$ on 
$N_{T}>30$ lattice with high statistics is necessary. However, we realized that $G_{\beta}\left(t_{n}\right)$ is very noisy[6], and we decided to start with a relatively smaller lattice of large statistics with some assumption for the spectral function $\rho(\omega)$.

The simplest non trivial from is a Bright-Wigner type proposed by Karsch and Wyld[7].

$$
\rho_{B W}(\omega)=\frac{A}{\pi}\left(\frac{\gamma}{(m-\omega)^{2}+\gamma^{2}}-\frac{\gamma}{(m+\omega)^{2}+\gamma^{2}}\right)
$$

In this case, shear viscosity is given by $\eta=4 A \gamma m /\left(\gamma^{2}+m^{2}\right)^{2}$. As this formula already has 3 parameters, in order to determine them by least squares, the lattice size in temperature direction $\left(N_{T}\right)$ should be $N_{T} \geq 8$. Then the minimum lattice size is $24^{3} \times 8$, to obtain a non trivial results.

Simulations are carried out by using the Iwasaki's improved action and standard Wilson action. The simulation points are at $\beta=3.05,3.3,4.5$ and 5.5 for improved action and $\beta=7.5$ and 8.5 for Wilson action. With roughly $10^{6} \mathrm{MC}$ measurements at each $\beta$, we determine the MatsubaraGreen's functions $G_{\beta}\left(t_{n}\right)$. Although the errors of the $G_{\beta}$ are still not small in large $t$ region, we can fit them with the spectral function $\rho$ give by Eq2.1, using a least square package SALS, and viscosities are calculated by these parameters. The errors are estimated by the jackknife method. The bin size of the jackknife analysis is taken to be $10^{6}$ and $2 \times 10^{6}$ for improved and Wilson actions, respectively.

The bulk viscosity is equal to zero within the range of error bars, while the shear viscosity remains fi nite. In order to determine the shear viscosity in physical unit, the lattice spacing $a(\beta)$ must be determined, because, in the lattice calculation, $\eta \times a^{3}$ is obtained. The relation between lattice spacing $a$ and $\beta$ is studied phenomenologically in $2.2 \leq \beta \leq 3.8$, and $5.58 \leq \beta \leq 6.5$ regions for improved[8] and Wilson actions [9], respectively. In the larger $\beta$ region, we adopt 2-loop renormalization group relation,

$$
a(\beta)=\frac{1}{\Lambda}\left(\frac{6 b_{0}}{\beta}\right)^{-\frac{b_{1}}{2 b_{0}^{2}}} \exp \left(\frac{-\beta}{12 b_{0}}\right),
$$

with $b_{0}=33 /\left(48 \pi^{2}\right)$, and $b_{1}=(34 / 3)\left(3 / 16 \pi^{2}\right)^{2}$. The $\Lambda$ is determined by the $a(\beta)$ at $\beta=3.8$ and 6.5 , and they result in $T_{c} / \Lambda=1.26$ and 47.35 for the improved and Wilson actions, respectively, where $T_{c}=270 \mathrm{MeV}$ in the quenched model. The results for the shear viscosity are shown in Fig. 1 with circle. It is seen that $\eta$ from both actions are consistent with each other.

\section{Comparison with perturbative results}

As we have calculated $\eta$ at rather high temperature regions, we compare our results with the perturbative results. The perturbative results are summarized as follows. The bulk viscosity, $\zeta$ is zero [5, 10]. The shear viscosity beyond the leading log is given by[11],

$$
\eta=\frac{\eta_{1} \cdot T^{3}}{g^{4}\left(\ln \left(\mu^{*} / g T\right)\right.}
$$

where $\eta_{1}=27.126$ and $\mu^{*} / T=2.765$, and $g$ is the running coupling constant. We use 2-loop formula for it,

$$
g^{-2}(T)=2 b_{0} \ln \left(\mu \frac{T}{T_{c}}\right)+\frac{b_{1}}{b_{0}} \ln \left(2 \ln \left(\mu \frac{T}{T_{c}}\right)\right)
$$




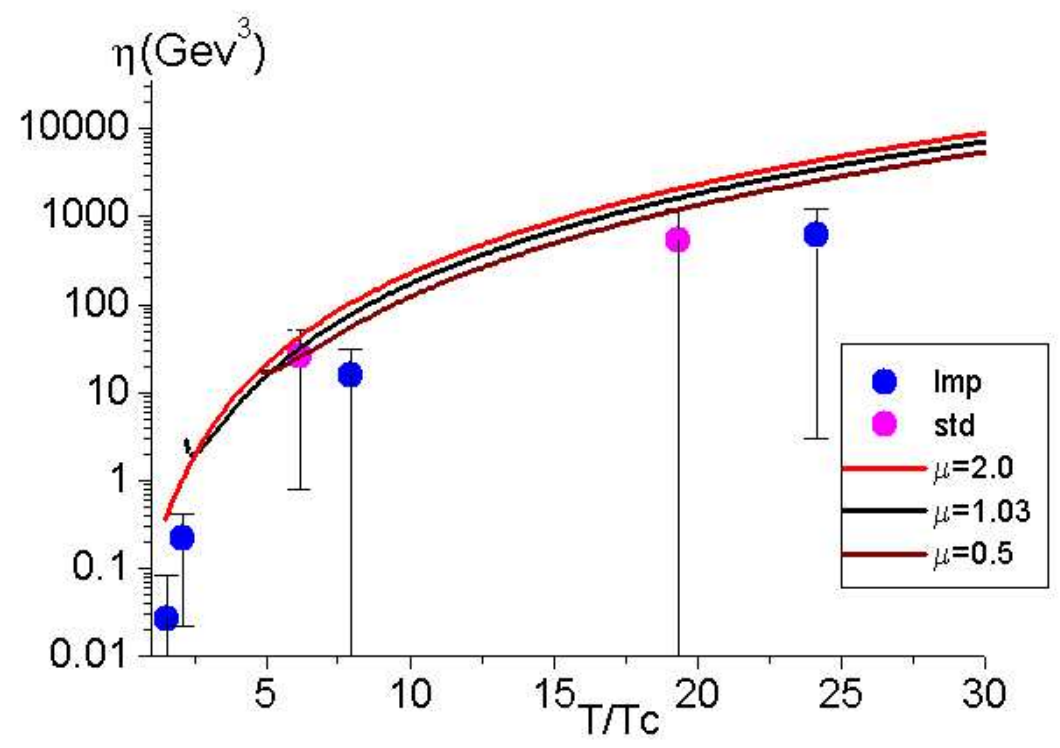

Figure 1: Shear viscosity in physical unit from lattice(circles) and perturbative calculations(lines). The lines show the ambiguity due to the choice of the scale parameter in two-loop running coupling formula.

Note that the perturbative approximations are renormalization-scale $\mu$ dependent. The simplest choice is $\mu=T_{c} / \Lambda_{Q C D} \sim 1.03$ [12]. However in order to see the ambiguity of the perturbative result due to $\mu$, we change $\mu$ in the range $0.5 \leq \mu \leq 2$. The results are also shown in Fig. 1 with line. In the large $T$ region, the $\mu$ dependence is not large. However at smallest $T$ of each line, it shows a small increase. It means the break down of the perturbative calculation. However until the break down starts the agreement of the perturbative and lattice results are satisfactory. Although our result depends on the assumption of the $\rho_{B W}$ given in Eq. 2.1, it may be a reasonable approximation for $d \rho / d \omega$ at $\omega=0$.

Let us proceed to the study of the $\eta / s$ ratio, recently discussed in [13]. The entropy on the lattice is reported by [8] and [14] in $T / T_{c}<4.5$, for improved and Wilson actions respectively. In the higher temperature region, where the data are not available, we use the high temperature limit values on $N_{T}=8$ lattice. The result is shown in Fig. 2 with circle. For the perturbative result on entropy, we use the formula of hard thermal loop calculation up to order $g^{3}[12]$. The results are also shown in Fig.2 2 with lines.

As explained in the Fig.11, the slight increase at the small $T$ region is observed for the perturbative results. The agreement between these two calculations are not good enough. However we do not think that it is a real discrepancy. Because if we decrease the scale parameter $\mu$, the agreement is improved. An important information from these results is the qualitative magnitude of the ratio $\eta / s$. In the $T / T c<3$ region, the ratio is small (0.1-0.4), where the perturbative results diverges. And for all the temperature regions we have studied, it is less than one. We don't think that it would become 10 times of present value, when the accurate determination of the spectral function is carried out. 


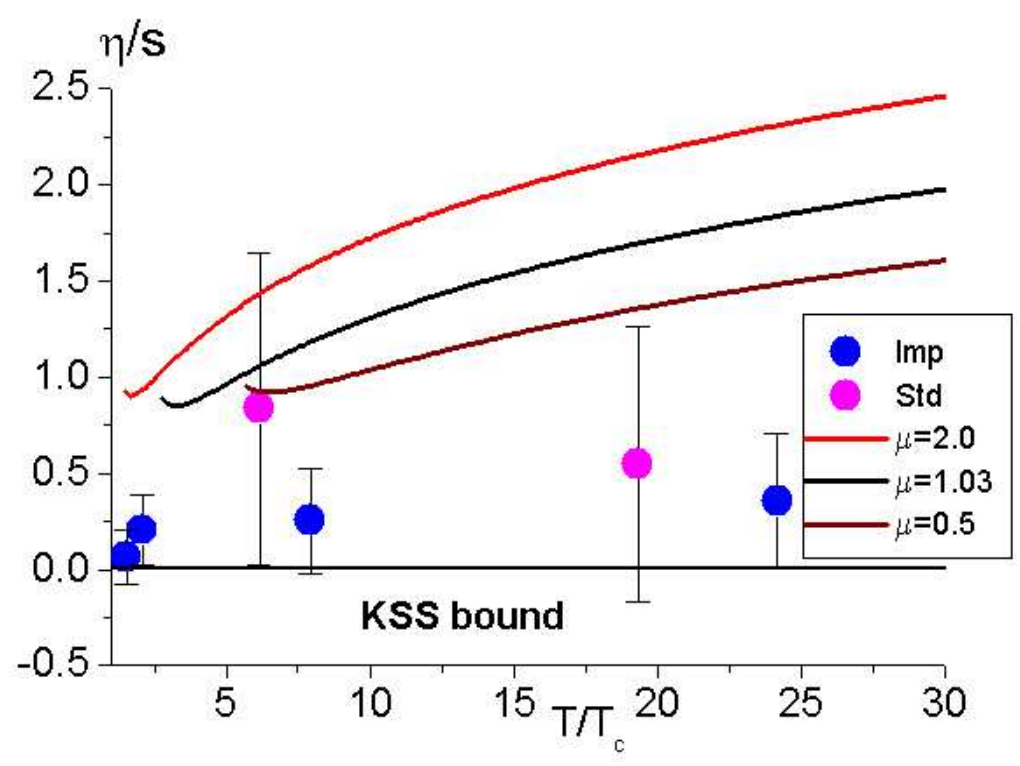

Figure 2: $\eta / s$ obtained by lattice simulations(circles) and perturbative calculations(lines). The $\mu$ is a scale parameter in the running coupling constant of Eq.3.2

\section{Discussions and conclusions}

\subsection{Fit of $G_{\beta}$ by other spectral functions}

G. Aarts and J.M.M. Resco have proposed an another form for the $\rho[3]$,

$$
\begin{gathered}
\rho(\omega)=\rho^{l o w}(\omega)+\rho^{h i g h}(\omega) \\
\frac{\rho^{l o w}(\omega)}{T^{4}}=x \frac{b_{1}+b_{2} x^{2}+\ldots}{1+c_{1} x^{2}+c_{2} x^{4}+. .}, \quad x=\frac{\omega}{T} \\
\rho^{h i g h}(\omega)=\theta(\omega-2 m) \frac{d_{A}\left(\omega^{2}-4 m^{2}\right)^{5 / 2}}{80 \pi^{2} \omega}[n(\omega / 2)+0.5]
\end{gathered}
$$

where $d_{A}=N_{c}^{2}-1$, and $n(\omega)=1 /(\exp (\omega / T)-1)$. We have three parameters to be determined, $m, b_{1}$ and $c_{1}$. The fi t by SALS could be made but $b_{1}<0$. We think that for these form of $\rho$, three parameters are too small to represent adequate spectral function.

For the next trial, we study effects of $\rho^{\text {high }}$ for the shear viscosity $\eta$. We assume $\rho$ is given by $\rho=\rho_{B W}+\rho^{\text {high }}$, where $\rho_{B W}$ is given by Eq. 2.1. By changing $m$, the change of $\eta$ is studied at $\beta=3.3$ for the improved action. When $\rho^{\text {high }}$ is absent $(m=\infty), \eta a^{3}=0.00225(201)$. If $m$ is set to be 5.0, 3.0 and 2.0, $\eta a^{3}$ becomes $0.00223(0.00191), 0.00194(0.00194)$ and 0.00126(0.00204), respectively. And at $m=1.8$, the contribution from $\rho^{\text {high }}$ becomes larger than the $G_{\beta}\left(t_{n}\right)$ of simulation at $t=1$, that fi $\mathrm{t}$ could not be done. Generally as $m$ decreases the contribution from $\rho^{\text {high }}$ increases and the $\rho$ in the small $\omega$ region is suppressed. In this case, it results in the decrease of $\eta$. 


\subsection{Conclusion and further studies}

On $24^{3} \times 8$ lattice, we have calculated the Matsubara-Green's function and determine the shear viscosity of gluon plasma. In the high temperature region, the agreement of the lattice and perturbative calculation is satisfactory. The lattice result on ratio $\eta / s$ in $T / T_{c} \leq 3$ is smaller than the extrapolation of the perturbative calculation and satisfi es the KSS bound.

Although our results depend on the form of the spectral function $\rho_{B W}$ given by Eq.2.1, we think that the qualitative features will not be changed. Because as discussed in the previous subsection, our results are stable if the high frequency part of the spectral function $\rho^{\text {high }}$ is included. We think that the $\eta$ and $\eta / s$ will not become 10 times of the present value when more accurate determination of the transport coeffi cients are carried out.

However it is important to carry out a more accurate calculation of the transport coeffi cients, independent of the assumption of the spectral function. For that goal, we are starting simulation on an anisotropic lattice, to apply the maximum entropy method.

\section{References}

[1] A. Hosoya, M. Sakagami and M. Takao,Nonequilibrium Thermodynamics in Field Theory: Transport Coefficients Annals of Phys. 154, 229(1984)

[2] A. Nakamura and S. Sakai,Transport Coefficients of Gluon Plasma Phys. Rev. Letters 94,072305(2005)

[3] G. Aarts and J.M.M. Resco, Transport Coefficients: spectral function and the lattice JHEP 4, 53(2002)

[4] D.N. Zubarev, Non-equilibrium statistical mechanics, Plenum, New York, 1974

[5] R. Horsley, W. Schoenmaker, Quantum Field Theories out of Thermal Equilibrium, (I).General considerations, (II). The transport coefficients for QCD Nucl. Phys. B280[FS18],716,735(1987)

[6] A. Nakamura, T. Saito and S. Sakai, Numerical Results for Transport Coefficients of Quark Gluon Plasma with Iwasaki's Improved Action Nucl. Phys. B (Proc. Suppl.) 63A-C,424(1998)

[7] F. Karsch and H.W. Wyld,Thermal Green's function and transport coefficients on the lattice Phys. Rev. D35, 2518(1987)

[8] M. Okamoto et al. Equation of state for pure SU(3) gauge theory with renormalization group improved action Phys. Rev.D60, 094510(1999).

[9] R.G. Edwards. U.M. Heller and T.R. Klassen Accurate scale determination for the Wilson gauge action Nucl. Phys.B517, 377(1998).

[10] A. Hosoya and K. Kajantie, Transport Coefficients of QCD Matter Nucl. Phys B250, 666(1985)

[11] P. Arnold, G.D. Moore and L.G. Yaffe, Transport coefficients in high temperature gauge theories: (II) Beyond leading log JHEP05 051(2003),[hep-ph/0302165]

[12] J-P. Blaizot, E. Iancu and A. Rebhan Entropy of QCD Plasma Phys. Rev. Letters83 2906(1999)

[13] P.K. Kovtun, D.T. Son and A.O.Starinets Viscosity in strongly interacting quantum field theories from black hole physics [hep-th/0405231]

[14] G. Boyd et al. Thermodynamics of SU(3) lattice gauge theory Nucl.Phys $\mathbf{B 4 6 9}$ 419(1996),[hep-lat/9602007] 